\title{
Le méloscepticisme des penseurs et écrivains européens : proposition de typologie
}

\section{Timothée Picard}

\section{Q OpenEdition}

\section{Journals}

\section{Édition électronique}

URL : http://journals.openedition.org/recherchestravaux/442

DOI : 10.4000/recherchestravaux.442

ISSN : 1969-6434

Éditeur

UGA Éditions/Université Grenoble Alpes

\section{Édition imprimée}

Date de publication : 15 mai 2011

Pagination : 13-36

ISBN : 978-2-84310-200-4

ISSN : 0151-1874

\section{Référence électronique}

Timothée Picard, « Le méloscepticisme des penseurs et écrivains européens : proposition de typologie », Recherches \& Travaux [En ligne], 78 | 2011, mis en ligne le 15 novembre 2012, consulté le 08 septembre 2020. URL : http://journals.openedition.org/recherchestravaux/442 ; DOI : https:// doi.org/10.4000/recherchestravaux.442 
Timothée PiCARD

Université Rennes 2

\section{Le méloscepticisme des penseurs et écrivains européens : proposition de typologie}

Il semble que, du XVII ${ }^{\mathrm{e}}$ au XXI ${ }^{\mathrm{e}}$ siècle, on puisse établir à gros traits une histoire de la «valeur musique» à travers la littérature et l'histoire des idées européennes. Ainsi, on constate la montée progressive de cette valeur à l'époque classique : la musique, d'abord placée au bas de la hiérarchie des arts, passe à son sommet avec le rousseauisme et le romantisme allemand. Elle trouve son âge d'or européen avec le symbolisme. Mais sa suprématie est remise en question par Mallarmé, Nietzsche, certaines avant-gardes européennes, puis, plus tard, par les philosophes partisans d'une esthétique analytique et non plus transcendantale. Depuis le nazisme et l'utilisation de la musique à des fins esthético-politiques, la tendance est au procès de la musique, ce qui n'empêche pas une certaine fascination de demeurer néanmoins. À ce sujet, notons que, pour plusieurs écrivains et penseurs, la musique est jugée non responsable de cette récupération, et, par effet de contrepoids, peut même être considérée comme un possible levier de rédemption; cependant que, pour d'autres, si une telle récupération a été pratiquée, c'est que, par nature, la musique la rendait possible.

Dans ce cadre, on peut distinguer, chez les écrivains et philosophes, quatre types de réticences à l'égard de la musique, que, pour plus de commodités, l'on appellera ici "méloscepticisme». Les écrivains et les philosophes peuvent d'abord se montrer mélosceptiques à une époque elle-même mélosceptique. C'est le cas, par exemple, des écrivains et hommes de lettres français à l'époque classique. Les écrivains et philosophes peuvent ensuite faire preuve d'une résistance mélosceptique à une époque éminemment mélomane - voire 
musicolâtre. Citons, entre autres, les cas de Kant à l'époque romantique, de Mallarmé ou de Tolstoï au moment du wagnérisme. Il faut encore évoquer le cas de figure particulier des écrivains et philosophes évidemment mélomanes, mais dont l'amour contrarié pour la musique a pu, ici et là, se transformer en haine apparente à son encontre. Nietzsche, Valéry, Bernhard ou Quignard sont des représentants exemplaires de cette tendance. Enfin, il faut effectuer un complément en forme de correctif : le méloscepticisme d'un écrivain ou philosophe peut concerner non pas la musique en général mais telle ou telle musique en particulier, et faire alors intervenir différents "modèles musicaux» de façon antagoniste et complémentaire. On fait ainsi jouer les unes contre les autres : musiques française, italienne ou allemande; musiques baroque, romantique ou moderne; musiques classique et jazz ou rock, etc.

Les discours contre la musique existent depuis l'Antiquité. Ils sont également présents dans les légendes orientales et les propos des Pères de l'Église. En toute logique, l'imaginaire mélosceptique moderne est très fortement imprégné par cette tradition. En ce qui concerne l'Antiquité, on peut par exemple citer ce propos de Thomas Mann, particulièrement représentatif:

Le mystère de la musique est grand - à la fois sensible et suprasensible par essence, alliant dans son étrange pouvoir combinatoire rigueur et rêve, morale et magie, raison et sentiment, nuit et jour, elle constitue sans doute le phénomène le plus profond, le plus inquiétant sur le plan philosophique, le plus fascinant de la culture et de l'humanité. [...] L'harmonie - c'est plus qu'un concept esthétique, c'est un principe cosmologique, et le mot remonte au début, ou presque de la pensée occidentale, il vient de la philosophie présocratique, de l'explication du monde de Pythagore. [...] Le nombre et le rapport numérique comme fondement constitutif de l'être - dans cette conception le Beau, l'Exact, le Raisonnable-Moral fusionnaient solennellement pour donner l'idée d'autorité qui animait la communauté pythagoricienne, l'école ésotérique de purification et de ressourcement religieux de la vie. Mais le monde n'est pas que belle ordonnance et harmonie des sphères, il contient des éléments irrationnels, démoniaques que la pensée grecque n’ignora jamais, mais inclut dans sa religiosité pour les y fondre. [...] Si l'univers est musique, la musique, elle, renvoie l'image du monde, du cosmos traversé de forces démoniaques. Elle est l'œuvre des nombres, service des nombres, calcul sacré, algèbre sonore. Mais les nombres eux-mêmes ne recèlent-ils pas un élément magique, quelque sorcellerie? [...] Ceux qui dirigent de la grande musique sont de par leur pratique professionnelle des éducateurs, et lorsqu'ils sont davantage que des spécialistes - ce qu'ils sont nécessairement quand ils sont grands - leur volonté pédagogique s'étend au domaine général de la morale, à la sphère humaine et politique ${ }^{\mathrm{r}}$.

I. Th. Mann, «La Mission de la musique. Pour les cinquante ans de direction d'orchestre de Bruno Walter" [1944], dans Etre écrivain allemand à notre époque, trad. D. Daun, Gallimard, coll. "Arcades», I996, p. 2I4-2I8. 
Les légendes orientales jouent par exemple un rôle essentiel dans Le Jeu des perles de verre de Hesse (Das Glasperlenspiel, 1943). Et, en ce qui concerne les Pères de l'Église, on peut renvoyer à l'augustinisme de Claudel, symptomatique d'une relation ambivalente à la musique, que résume au mieux, à travers son œuvre, la coprésence de ces deux injonctions paradoxales: "Cave musicam» ("Défie-toi de la musique!») et "Non impedias musicam» («N'empêche pas la musique!») 2. Enfin, ces trois sources d'influence se retrouvent par exemple dans un ouvrage qui, à cet égard, semble prendre une valeur paradigmatique : La Haine de la musique de Pascal Quignard (1996).

À travers ces discours sont toujours plus ou moins en jeu trois domaines d'incertitudes et de débats au sujet de la musique. Est d'abord en question la capacité ou non de la musique à représenter et à signifier. En lien direct avec cette première donnée, on s'interroge ensuite sur le lien éventuel que la musique, plus que tout autre art, entretiendrait avec un quelconque infini. Enfin, on glose ses effets physiologiques et moraux sur l'individu et la collectivité, et leur supposée ambiguïté. Ces sujets d'incertitudes et de débats, d'ordre général, trouvent cependant une résonance particulière dans le cas de l'écrivain qui, régulièrement, exprime le sentiment que la musique pose un défi au langage. Il s'agit, en premier lieu, d'un défi d'ordre intersémiotique, celui de dire la musique et de produire une jouissance d'intensité comparable. Mais s'ajoute en deuxième lieu un défi d'ordre métaphysique, qui consiste à exprimer le désir de prendre en charge, dans le langage, cet infini avec lequel la musique semble entretenir un rapport privilégié. Notons enfin que ce défi, né avec le rousseauisme et le romantisme allemand, peut être remis en question dès la fin du romantisme, et se transformer alors en défi éthique, celui qui découle des effets bénéfiques ou maléfiques que la musique aurait sur l'individu et la collectivité - sorte de défi auquel l'histoire semble apporter un certain crédit. Ce triple défi est à l'origine de la plupart des manifestations de réticence ou de haine que les écrivains formulent à l'égard de la musique. Précisons toutefois que, la plupart du temps, il engage certes en un sens la musique, mais aussi - et surtout - l'écriture et la littérature. Par un tel récit, les écrivains semblent en effet particulièrement désireux de cerner leurs limites, d'envisager un programme de reconquête, et surtout de les amener à se rénover.

2. Voir P. Claudel, Mes idées sur le Théâtre [1966], Gallimard, I993, p. 6I et Mémoires improvisés [1954], recueillis par J. Amrouche, Gallimard, coll. "Les Cahiers de la NRF", 200I, p. 309. 


\section{Écrivains mélosceptiques à une époque mélosceptique}

Avec les exemples de la France à l'époque classique, puis des avant-gardes européennes de la première moitié du $\mathrm{xx}^{\mathrm{e}}$ siècle, envisageons tout d'abord le cas des écrivains mélosceptiques à une époque elle-même mélosceptique.

Dans le cas de la France à l'époque classique, rappelons tout d'abord que la musique est alors soumise à deux critères discriminants, la mimésis d'une part et le modèle rhétorique de l'autre, et qu'en raison de ses supposées carences sur ces deux plans, elle figure au bas de la hiérarchie des arts - et est même parfois violemment rejetée. En ce qui concerne la mimésis, le problème en question est en effet que la musique semble moins apte à imiter que les autres arts. D'Alembert écrit par exemple dans le discours préliminaire de l'Encyclopédie :

À la tête des connaissances qui consistent dans l'imitation, doivent être placées la peinture et la sculpture, parce que ce sont celles de toutes où l'imitation approche le plus des objets qu'elle représente, et parle le plus directement à l'âmeje

Et, pour ce qui engage le second critère, rappelons que la musique n'est envisageable à l'époque - c'est-à-dire considérée sinon comme légitime et pertinente du moins comme tolérable - qu'à la condition qu'elle soit soumise à un modèle rhétorique. On dit en effet qu'elle doit construire une espèce de discours, et que plus elle entre en rapport étroit avec un texte, meilleure elle est. Symétriquement, en raison de son apparente insignifiance, la musique pure est dès lors potentiellement considérée comme monstrueuse. C'est tout le sens de cette fameuse anecdote - probablement apocryphe, mais reconduite de texte en texte - mettant en scène le philosophe Fontenelle qui, lors d'un concert de musique instrumentale, se serait soudain levé furieux et, levant le poing, se serait écrié : «Sonate, que me veux-tu?». Dès lors, pour pallier ce manque, on invite le musicien à avoir en permanence à l'esprit une sorte de récit lorsqu'il compose. À ce sujet, D’Alembert écrit par exemple :

Toute cette musique purement instrumentale, sans dessein et sans objet, ne parle ni à l'esprit, ni à l'âme et mérite qu'on lui demande avec Fontenelle : Sonate, que me veux-tu? Les auteurs qui composent de la musique instrumentale ne feront qu'un vain bruit, tant qu'ils n'auront pas dans la tête, à l'exemple, dit-on, du célèbre Tartini, une action ou une expression à peindre4.

3. D’Alembert, Discours préliminaire de l'Encyclopédie, p. XI, cité par B. Cannone, Philosophies de la musique: 1752-1789, Aux amateurs de livres, 1990, p. 98.

4. Id., Euvres philosophiques, historiques et littéraires, Paris, Bastien, I805, t. III, "De la liberté de la musique», p. 403, cité par V. Anger, Le Sens de la musique, I750-I9oo, Éditions Rue d'Ulm-ENS, 2005, p. 46-47. 
Cette conception d'ensemble a des conséquences plus directes sur le genre de l'opéra. Pour ses plus vifs détracteurs, la musique nuirait en effet à la poésie dans l'œuvre d'art mixte : elle desservirait la compréhension et, par conséquent, le plaisir que celle-ci pourrait procurer. Si on condamne l'opéra, c'est aussi parce que la musique nimberait le langage de sensualité, et engendrerait nécessairement une simplification de la pensée. Dès lors, cette forme d'art est considérée comme structurellement immorale : la raison y est nécessairement endormie au profit des passions, devenues le territoire propre de la musique; et, parmi les passions, c'est l'amour qui dominerait, et deviendrait dès lors le thème essentiel du genre opéra. En la matière, la position de Bossuet, dans ses Maximes et réflexions sur la comédie (I694), est tout à fait exemplaire :

Si Lulli a excellé dans son art, il a dû proportionner, comme il a fait, les accents de ses chanteurs et de ses chanteuses à leurs récits et à leurs vers : et ses airs, tant répétés dans le monde, ne servent qu’à insinuer les passions les plus décevantes, en les rendant les plus agréables et les plus vives qu'on peut. Il ne sert de rien de répondre qu'on n'est occupé que du chant et du spectacle, sans songer au sens des paroles, ni aux sentiments qu'elles expriment; car c'est là précisément le danger, que pendant qu'on est enchanté par la douceur de la mélodie, ou étourdi par le merveilleux du spectacle, ces sentiments s'insinuent sans qu'on y pense, et gagnent le cœur sans être aperçus 5

Pour les autres, moins catégoriques, la musique ne saurait de toutes les façons se passer de poésie : c'est que la première présenterait l'avantage de "fixer" ce que la seconde pourrait avoir de trop imprécis. Dès lors, la musique aurait pour principale qualité d'amplifier le charme de la poésie. Enfin il va de soi que, dans une telle conception de la musique, la parole a incontestablement une prévalence sur la musique.

Voyons maintenant ce qu'il en est pour les avant-gardes européennes de la première moitié du $\mathrm{xx}^{\mathrm{e}}$ siècle. Une partie de l'expressionnisme allemand mis à part, on peut en effet avancer que, globalement, les avant-gardes du début du $\mathrm{Xx}^{\mathrm{e}}$ siècle sont initiatrices d'un changement de paradigme, tendant à remplacer, en littérature comme ailleurs, le modèle musical par le modèle pictural. Julien Gracq explique bien ce processus dans En lisant en écrivant (1980) :

Il y a pour chaque époque une hégémonie mal avouée, mais effective, qui passe d'un art à l'autre capricieusement. Il est difficile, par exemple, de se faire une idée de la montée foudroyante, en influence et en prestige, de la peinture à l'époque d'Apollinaire, alors que, vingt ans auparavant, Wagner avait littéralement écrasé le symbolisme sous sa masse sonore ${ }^{6}$.

5. Bossuet, "Lettre au père Caffaro", précède Maximes et réflexions sur la comédie (I694), cité par C. Kintzler, Poétique de l'opéra français de Corneille à Rousseau, Minerve, I99I, p. 3.

6. J. Gracq, En lisant en écrivant, Corti, 1980, p. 279-280. 
Parmi les chefs de file des avant-gardes, on recense ainsi de francs mélosceptiques tels que Marinetti (voir ses textes contre le wagnérisme et le tango) et surtout Breton. Dans Le Surréalisme et la Peinture (1928), celui-ci écrit on ne peut plus clairement :

Qu'il me soit permis d'accorder à l'expression plastique une valeur que par contre je ne cesserai de refuser à l'expression musicale, celle-ci de toutes la plus profondément confusionnelle. En effet les images auditives le cèdent aux images visuelles non seulement en netteté, mais encore en rigueur et, n'en déplaise à quelques mélomanes, elles ne sont pas faites pour fortifier l'idée de la grandeur humaine ${ }^{7}$.

Sans la rejeter complètement, Brecht s'attaque de son côté au caractère hypnotisant, jouissif et donc mystificateur de la musique. Se retournant contre l'héritage du romantisme allemand, il refuse en tous les cas qu'on la place au sommet de la hiérarchie des arts.

\section{Résistances mélosceptiques à une époque musicolâtre}

Il nous faut maintenant considérer quelques cas exemplaires de résistances mélosceptiques à une époque musicolâtre. En effet, si la musique passe au sommet de la hiérarchie des arts avec le rousseauisme et le romantisme allemand, quelques réticences demeurent cependant. C'est notamment le cas pour des figures à cheval sur le classicisme et le romantisme telles que Kant.

Dans le paragraphe 53 de la Critique de la faculté de juger, Kant propose deux systèmes parallèles de hiérarchie des arts. Dans le premier système qui se construit selon le critère de la culture, la musique occupe la dernière place, loin derrière les arts figuratifs. C'est que, pour Kant, les arts figuratifs sont plus sérieux que la musique car ils procurent des sensations durables. Dans la mesure où ils relient concepts et sensibilité, ils apportent de la matière à l'entendement, et donnent ainsi une urbanité aux facultés de connaître. À l'opposé, la musique n'est pas urbaine car elle s'impose à qui ne veut pas l'entendre, et restreint la possibilité de dialoguer librement - condition sine qua non de l'urbanité. Mais, dans un système établi selon le critère de la jouissance, en laquelle Kant voit tout de même un instrument d'universalité, la musique occupe cette fois la deuxième place après la poésie. C'est qu'à la différence de la poésie, la musique ne serait faite que de sensations pures sans

7. A. Breton, «Le surréalisme et la peinture» dans Le Surréalisme et la Peinture, Gallimard, 1965, p. II. Sur la question des liens complexes que le surréalisme a entretenus avec la musique, on lira avec profit: S. Arfouilloux, Que la nuit tombe sur l'orchestre : surréalisme et musique, Fayard, 2009. 
concept et ne laisserait pas de place à la réflexion. Il s'agirait d'une langue universelle, certes, intelligible à tout homme, mais qui reviendrait purement et simplement à "composer» des sensations, et non pas à articuler sensibilité et concepts, comme le fait le langage. C'est pourquoi elle exigerait non pas continuité et stabilité, qui sont le propre de l'entendement, mais au contraire, pour alimenter les besoins de la seule sensibilité, inconstance et changement. De façon plus générale, de Kant à Hegel en passant par Goethe ${ }^{8}$, il faut rappeler qu'il existe, au sein du romantisme allemand, une poche de résistance logocentrique et mélosceptique, que Nietzsche, à la fin du siècle, réactive de façon personnelle et ambivalente. Même le très mélomane Kierkegaard semble adopter sur le sujet une position similaire. Le philosophe danois voit en effet dans cette génialité sensible qui caractérise la musique, dans son caractère indéterminé et indéterminable, les signes de la grandeur et de la faiblesse de la musique par rapport au langage. Liée à l'érotique, il ne la place dans son parcours spirituel type qu'à un stade esthétique, et non pas éthique ou religieux'.

À la fin du siècle, plusieurs fictions et essais de Tolstö̈ donnent également une image particulièrement négative de la musique. La Sonate à Kreutzer s'en prend à Beethoven, certaines scènes d'Anna Karénine ou de La Guerre et la Paix à l'opéra, et les essais regroupés sous le titre Qu'est-ce que l'art? à Wagner. Si, dans ces textes, l'écrivain ne semble à première vue s'attaquer qu'à quelques musiques particulières, les propos tenus sont parfois accompagnés de telles extrapolations qu'il semble que toute la musique moderne soit finalement concernée - quand ce n'est pas toute musique et tout art occidental : occasion pour lui d'opérer par métonymie un véritable procès de civilisation. Ainsi, dans ses essais contre Wagner, Tolstoï fait remonter très loin en amont l'origine de la décadence dont Wagner serait le symptôme et la synthèse. Autour de lui s'étoilent, entre autres, les figures de Goethe, Mallarmé, Ibsen, Kipling, Huysmans, Zola, Beethoven, Liszt, Berlioz, Brahms, Strauss, et Delacroix. Puis l'anathème tombe sur tous les fondements de la culture occidentale : les tragiques grecs, Dante, Le Tasse, Milton, Shakespeare, Bach, etc. Est en cause, selon Tolstoï, l'effet moralement dévastateur de la musique et de la fiction opératique, envisagées comme des mensonges, des illusions, bien propres à exaspérer la sensibilité nerveuse de l'auditeur, à menacer l'intégrité

8. En dépit, pour Hegel, des propos élogieux sur Mozart, Gluck et l'opéra italien et, pour Goethe, des éloges de Bach et Mozart, et de la proximité avec Reichardt. Sur Hegel et la musique, voir A. P. Olivier, Hegel et la musique : de l'expérience esthétique à la spéculation philosophique, Honoré Champion, 2003.

9. Voir notamment $S$. Kierkegaard, "Les étapes érotiques spontanées ou l'érotisme musical» dans Ou bien... ou bien [1843], Gallimard, 1943, p. 39-106. 
du sujet, et à le préparer aux passions et aux actes les plus dévastateurs. L'une et l'autre relèveraient souvent du plaisir pour élite oisive et décadente, contre lequel le peuple authentique doit absolument se prémunir. Le long extrait suivant, tiré de La Sonate à Kreutzer, permet de se faire une idée précise du méloscepticisme de Tolstoï :

Ils jouaient La Sonate à Kreutzer de Beethoven. Connaissez-vous le premier presto? Vous le connaissez? s'écria-t-il. Ah! Quelle chose terrible que cette sonate! Surtout ce mouvement-là. Et, en général, quelle chose terrible que la musique! Qu'est-ce exactement? Je ne le saisis pas. Qu'est-ce que la musique? Quelle est son action? Et pourquoi agit-elle comme elle le fait? On dit que la musique agit de façon à élever l'âme... quelle stupidité, quel mensonge! Elle agit, elle agit terriblement, je parle pour moi, mais nullement de façon à élever l'âme, ni de façon à l'abaisser, mais de façon à l'exaspérer. Comment vous dire? La musique m’oblige à m'oublier, à oublier ma vraie condition, elle me transporte dans un état qui n'est pas le mien; sous l'influence de la musique, j'ai l'impression que je sens ce qu'en réalité je ne sens pas, que je comprends ce que je ne comprends pas, que je peux ce que je ne peux pas. J'explique cela en disant que la musique agit comme le bâillement, comme le rire; je n'ai pas sommeil, mais je bâille en voyant quelqu'un bâiller; je n'ai aucune raison de rire, mais je ris en entendant quelqu'un rire. La musique, elle, me transporte d'emblée, immédiatement, dans l'état d'âme où se trouvait celui qui l'a écrite. Je confonds mon âme avec la sienne et avec lui je passe d'un état à un autre, mais pourquoi je fais cela, je l'ignore. L'homme qui a écrit mettons La Sonate à Kreutzer, Beethoven, savait pourquoi il se trouvait dans cet état qui l'a porté à certains actes, aussi pour lui cet état a une signification, pour moi aucune. C'est pourquoi la musique ne fait qu'irriter, elle ne conclut pas. Si par exemple on joue une marche militaire, les soldats défilent à son rythme et la musique a atteint son but; on a joué une danse, j'ai dansé pendant ce temps-là, la musique a atteint son but; on a chanté une messe, j'ai communié, la musique a encore atteint son but; autrement ce n'est qu'une surexcitation et qu'y a-t-il à faire au sein de cette surexcitation? Rien. C'est pourquoi la musique exerce parfois une action si terrible, si redoutable. En Chine, la musique est l'affaire de l'État. C'est ainsi que cela doit être. Peut-on admettre que n'importe qui puisse à son gré hypnotiser seul une ou plusieurs personnes et ensuite faire d'elles ce qu'il veut? Et surtout que cet hypnotiseur soit le premier homme de mauvaises mœurs rencontré en chemin? Et entre les mains de qui est tombé ce redoutable instrument? Prenons par exemple cette Sonate à Kreutzer, le premier presto. Peut-on jouer ce presto dans un salon parmi des femmes décolletées? Le jouer, puis applaudir, manger des glaces et se raconter le dernier potin? Ces morceaux-là, on ne devrait les jouer qu'en certaines circonstances importantes, graves, et lorsqu'on est requis d'accomplir certains actes importants, graves, en harmonie avec cette musique. Autrement, cet appel inopportun à une énergie, à des sentiments qui n'ont pas lieu de se manifester, ne peut avoir qu'un résultat néfaste. Sur moi, du moins, cela produit un effet désastreux; c'était comme si des sentiments que je croyais tout à fait nouveaux, des possibilités que j'ignorais jusqu'alors se révélaient à moi ${ }^{\mathrm{I}}$.

Io. L. Tolstoï, La Sonate à Kreutzer [Gallimard, I958], Le Livre de Poche, I97I, p. II3. 
On le sait, à la fin du XIX ${ }^{\mathrm{e}}$ siècle, alors que le symbolisme wagnérolâtre bat son plein, Mallarmé fait peu à peu dissidence et, par là, inaugure la remise en question d'un modèle musical devenu omniprésent en littérature. La relation de Mallarmé à la musique est en effet celle d'un mélosceptique et d'un logocentrique patenté. Pour autant, s'exprimant tout à la fois sous la forme d'une attraction et d'une répulsion, sa figure nous place d'ores et déjà à la frontière de notre prochain cas de figure. En effet, à l'égard de la musique, Mallarmé commence par reprendre à son compte les arguments positifs qui étaient ceux du romantisme allemand et qui, désormais, sont ceux du symbolisme : en raison de sa nature hiéroglyphique, la musique serait un langage qui, malgré l'invasion du prosaïsme philistin, parviendrait à conserver son mystère non profané; elle serait par excellence l'art de la suggestion, et, avec le mythe et le théâtre, le levier par lequel un peuple pourrait accéder à son "type», à la belle image de lui-même. Dans Crayonné au Théâtre, il écrit ainsi que la musique permet de se

[...] replonger, de quelque part, dans le peuple [...] au sein de la Passion de l'Homme ici libéré selon quelque source mélodique naïve. Pareil emploi de la Musique la tient prépondérante comme magicienne, attendu qu'elle emmêle et rompt ou conduit un fil divinatoire $[\ldots]^{\mathrm{II}}$

Cependant, en tant que telle, la musique paraît à Mallarmé insuffisante, voire dangereuse. La musique serait pour lui quelque chose comme une onde muette, à laquelle ferait défaut la bouche qui profere et donne un sens. "L'éruptif multiple sursautement de la clarté» propre à la musique, écrit-il, est «vain, si le langage, par la retrempe et l'essor purifiants du chant, n'y confere un sens ${ }^{12}{ }^{2}$. Malgré l'éloge premier, l'ambivalence du rapport du poète à la musique, et les mérites supérieurs qu'il confere au langage, apparaissent dès lors avec évidence - par exemple dans ce propos, tiré du "Mystère dans les Lettres»:

Les déchirures suprêmes instrumentales, conséquences d'enroulements transitoires, éclatent plus véridiques, à même, en argumentation de lumière, qu'aucun raisonnement tenu jamais; on s'interroge, par quels termes du vocabulaire sinon dans l'idée, écoutant, les traduire, à cause de cette vertu incomparable. [...] Lécrit, envol tacite d'abstraction, reprend ses droits en face de la chute des sons nus ${ }^{13}$.

Le langage, qui est la face radieuse de l'Idée, est là pour faire ressortir l'éclat fugace de la musique, sa part d'ombre. Tel est «l'indéracinable préjugé d'écrivain »

II. S. Mallarmé, "Crayonné au théâtre», dans Crayonné au théâtre, CEuvres complètes, Gallimard, coll. «Bibliothèque de la Pléiade», I945, p. 297.

I2. Id., "La musique et les lettres", ibid., p. 648.

I3. Id., "Le mystère dans les lettres", ibid., p. 385. 
dont ne se défera jamais Mallarmé à l'égard de la musique, et qui consacre donc à ses yeux le triomphe modeste de la Poésie:

Je me figure par un indéracinable sans doute préjugé d'écrivain, que rien ne demeure sans être proféré; que nous en sommes là, précisément, à rechercher, devant une brisure des grands rythmes littéraires [...] et leur éparpillement en frissons articulés proches de l'instrumentation, un art d'achever la transposition, au Livre, de la symphonie ou uniment de reprendre notre bien : car, ce n'est pas de sonorités élémentaires par les cuivres, les cordes, les bois, indéniablement mais de l'intellectuelle parole à son apogée que doit avec plénitude et évidence, résulter, en tant que l'ensemble des rapports existant dans tout, la Musique ${ }^{14}$.

Car, pour Mallarmé, le défi que la musique porte à la littérature se surmonte dans cette idée que l'accomplissement de l'idéalité musicale se fera finalement avant tout dans un cadre littéraire et non pas musical. Au demeurant, pour Mallarmé, la musique est de moins en moins l'art des sons au sens strict, quand bien même celui-ci peut être vanté, mais plutôt, conformément à ce que le mot signifiait à son origine, une certaine façon de composer l'œuvre, et d'en agencer les éléments. Ce que, finalement, et paradoxalement, la poésie parviendrait mieux que la musique à mettre en œuvre. Mais, pour ce faire, il faut qu'à son tour le langage devienne instrument :

Tout, la polyphonie magnifique instrumentale, le vivant geste ou les voix des personnages et des dieux au surplus un excès apporté à la décoration matérielle, nous le considérons dans le triomphe du génie, avec Wagner, éblouis par une telle cohésion, ou un art, qui aujourd'hui devient la poésie : or va-t-il se faire que le traditionnel écrivain de vers, celui qui s'en tient aux artifices humbles et sacrés de la parole, tente, selon sa ressource unique subtilement élue, de rivaliser! Oui, en tant qu'un opéra sans accompagnement ni chant, mais parlé; maintenant le livre essaiera de suffire, pour entr'ouvrir la scène intérieure et en chuchoter les échos ${ }^{15}$.

Alors seulement «la Poésie, proche l'idée, est Musique, par excellence ${ }^{\mathrm{I6}}$ ».

\section{L'amour-haine de la musique chez les écrivains et philosophes mélomanes}

Après avoir considéré le méloscepticisme propre à telle ou telle figure dans des époques elles-mêmes mélosceptiques, puis le méloscepticisme exemplaire de quelques figures dissidentes dans des moments musicolâtres, il nous faut maintenant envisager le cas probablement le plus intéressant : celui de

I4. Id., "Crise de vers", ibid., p. 368.

I5. Id., "Planches et feuillets", ibid., p. 328.

I6. Id., «Le livre, instrument spirituel», dans Variations sur un sujet, ibid., p. 38I. 
l'amour-haine de la musique formulé par les écrivains et philosophes les plus évidemment mélomanes. À partir d'un sentiment plus ou moins fort de défi lancé par la musique à l'être de l'écrivain mélomane en général, à l'entreprise d'écriture en particulier, l'axiologie de l'écrivain mélomane peut en effet balancer entre le "mélocentrisme», c'est-à-dire la célébration de la sphère musicale aux dépens de la littérature et du langage, et le «logocentrisme», autrement dit, à l'inverse, la louange de la sphère littéraire et du langage aux dépens de la musique. Envisageons ici quelques-unes des figures les plus représentatives.

La relation que Valéry, héritier de Mallarmé, entretient avec la musique, placée sous le signe de la notion de «limite», est partagée entre l'admiration, le désespoir stérilisant, et l'émulation féconde. Voyons d'abord ce qu'il en est de la fascination. La relation privilégiée que Valéry a entretenue, durant ses années de formation, avec la musique, a pris la forme d'une tentative de compréhension de ce qui fait la spécificité stupéfiante de l'expérience musicale. Les caractéristiques de la musique constituent en effet pour Valéry sinon des valeurs, du moins des objectifs à atteindre en tant que créateur. Une des constantes par lesquelles Valéry rend compte du caractère décisif de la création artistique est la notion de "maximum». Wagner, par exemple, en réussissant hyperboliquement à transformer l'extrême passion de l'intuition artistique en ferment énergétique d'une création parfaitement dominée, aurait ainsi atteint, selon Valéry, un "maximum». Fascination, donc, mais qui est doublée d'un certain désespoir. L'expérience wagnérienne et l'analyse à laquelle elle donne lieu confrontent en effet peu à peu Valéry à un impossible : les thèmes du désespoir, de l'impuissance, engendrés par la musique, et, en corollaire, celui, en forme de spectre, du renoncement à la littérature, prennent alors une place croissante dans ses écrits. Sa fascination, admirative jusqu'à l'écrasement, tient en effet à ce que la musique semble concilier les polarités a priori les plus antagonistes de l'être humain. Or Valéry fait précisément sur le plan existentiel l'expérience douloureuse de ce que ces dichotomies ont d'irréductible, et constate que le langage ne fait que les entériner et même les renforcer.

S'initie alors un retournement. Pour ne pas verser dans la procrastination, Valéry se met en effet à développer une sorte de «logocentrisme de protection" Il redit la suprématie que, par certains aspects, le langage manifesterait sur la musique. Au sein de ce "logocentrisme mélosceptique», on trouve plusieurs arguments caractéristiques. En matière de plaisir esthétique, Valéry déclare ainsi préférer, à de certains moments, la modération de la littérature à la violence de la musique. Il dit aussi refuser le dispositif de manipulation individuelle et collective que mettrait selon lui en place la musique. 
Il dénonce une forme d'assuétude à la jouissance musicale qui, selon lui, serait comparable à une dépendance au sexe et à la drogue. Et, tout comme Mallarmé, il s'en prend au fait que la musique, bouche d'ombre pré- ou infralangagière, n'est pas capable de donner forme à l'idée dans toute sa clarté. Toute cette réflexion va engager, chez Valéry, un programme de reconquête, au sein duquel la musique continue cependant d'être l'interlocutrice et le modèle herméneutique privilégiés ${ }^{17}$.

En plus de l'influence de Mallarmé, celle exercée par Nietzsche sur Valéry est évidente. On le sait, la mélomanie de Nietzsche est entièrement soumise à la dialectique de la fascination et de la réticence. Notons que cette dialectique peut en outre s'articuler à une «imagologie»: le mélocentrisme est en effet considéré par Nietzsche comme une caractéristique de l'italianité, puis de la germanité, et cela, contre le logocentrisme, qui, lui, est considéré comme le fait de la francité. Pendant tout le temps où le philosophe se déclare favorable à Wagner et à tout ce qu'il représente, Nietzsche considère que, face aux méfaits du «socratisme civilisationnel» - cette réduction de l'être par l'effet de l'inflation théorique et le culte de l'apparence apollinienne -, la seule sphère dans laquelle la pratique philosophique est préservée est l'esthétique. Dans ce cadre, la musique seule, associée au mythe et à la poésie, permettrait que l'"état d'âme tragique», la plus petite définition ontologique du genre humain, "ne périsse pas». La musique, proche du monde dionysiaque, est alors considérée comme la manifestation même de la germanité, et Wagner en est l'incarnation par excellence. Après s'être retourné contre Wagner, Nietzsche s'en prend aux caractéristiques de la musique du compositeur, synthèse, selon lui, de deux données certes hétérogènes d'un point de vue imagologique, mais dont il instruit cependant un égal procès : la germanité, d'une part, et le symbolisme, d'origine française et rapidement cosmopolite d'autre part. À travers ces deux entités, il s’attaque à une musique de la profondeur nordique (romantique, chrétienne, métaphysique, etc.) et met en avant les ambiguïtés de la modernité (attirance pour le mélange des arts, perversion de la musique par la littérature, histrionisme propre à la société théâtrocratique, etc.) qui l'apparentent selon lui à une décadence. Contre la sphère nordique et son "mythos», il réactive les caractéristiques de la sphère méditerranéenne et de son "logos», autrefois décriés. Et contre la musique dionysiaque, il dresse l'idéal apollinien du moralisme français, et pratique le discours clinique à caractère pseudo-scientifique.

17. Sur ce point, nous renvoyons à notre étude "Valéry et l'investigation du Moi musical» dans T. Picard, L'art total, grandeur et misère d'une utopie (autour de Wagner), Presses universitaires de Rennes, 2006, p. 3II-3I6. 
La posture antimusicale peut alors engager non plus la seule musique allemande, mais la musique en général. Paradoxalement, celle-ci se trouve désormais associée aux deux pôles antagonistes de la dialectique nietzschéenne : elle peut être considérée comme à la fois caractéristique de la lourdeur germanique (autrement dit placée du côté de la résistance de la "culture» contre la "civilisation") et des méfaits de la démocratie moderne (c'est-à-dire alors plutôt du côté inverse : celui de la "civilisation» contre la "culture») - sachant que Nietzsche peut être amené à effectuer la critique tantôt de l'un tantôt de l'autre pôle. Ainsi, dans le premier cas, la musique, qualifiée de «tard venue de la civilisation", est considérée comme une dernière réaction contre la philosophie des Lumières. On retrouve alors certains des propos de Kant : la musique s'opposerait à la parole, au dialogue, à la dialectique, à la discursivité; elle ne serait chez elle que là où les hommes ne peuvent et ne doivent discuter; parce qu'elle peut dire en même temps le vrai et le faux, elle ne souffrirait aucune pondération, aucune réfutation. Reportée sur l'Allemagne, une telle musique montrerait l'échec de l'Allemagne, une Allemagne de la pesanteur, de l'absence ou de l'excès de style, une Allemagne de l'avant-hier et de l'après-demain, mais pas de cet aujourd'hui, qui est le temps de Zarathoustra. En même temps, et ce sont alors des arguments qui appartiennent au second cas, elle serait capable de sceller le triomphe de tous ces idéaux de la civilisation que Nietzsche abhorre : la révolution, le peuple, la démocratie, etc. Désormais, musique allemande et européenne, en principe antithétiques, deviendraient donc équivalentes, toutes deux absorbées par une même chimère humaniste et supranationale. Notons toutefois que ce discours doublement antimusical n'empêche pas Nietzsche de continuer à utiliser le champ lexical de la musique pour caractériser l'activité et l'écriture philosophique à ses yeux idéales ${ }^{\mathrm{r}}$.

Thomas Mann hérite quant à lui très évidemment du dispositif nietzschéen, et effectue un parcours similaire à celui de son maître - complexité de la dialectique comprise. Ce parcours ne se déroule toutefois plus de façon abstraite : il est désormais motivé par l'histoire. Avant 1922, et l'adhésion à la République de Weimar, la pensée de Thomas Mann est d'ordre nationaliste. Dans ce texte capital que sont les Considérations d'un apolitique, il définit le monde de la «culture» (la sphère germanique) contre le monde de la «civilisation» (la sphère méditerranéenne) en faisant de la musique un critère déterminant. À ce titre, et pour bien déclarer son hostilité à la civilisation, dont l'art de prédilection serait selon lui la littérature, Thomas Mann avance la proposition encyclopédique Wagner (T. Picard [dir.]) Actes Sud - Cité de la Musique, 20IO, p. I428-I432. 
paradoxale selon laquelle ses œuvres ne seraient pas des romans mais des partitions. La lecture qu'il fait de son œuvre passée est alors marquée par la surdétermination de leur supposée nature musicale - une caractéristique qui, selon lui, engagerait non seulement la substance mais encore la forme de ses œuvres, qu'il place tantôt sous le signe de la fugue et du contrepoint de Bach, tantôt sous celui du leitmotiv wagnérien. Ajoutons encore qu'à cette époque l'hypothèse d'une essence musicale de la germanité est, chez l'écrivain, mise en rapport avec une autre caractéristique supposée de cette dernière, l' "apolitisme» (par là, il faut entendre le peu de sens et de sympathie dont feraient preuve les Allemands à l'égard du politique), et que ces deux données sont d'ailleurs considérées par lui comme étant de quasi synonymes. Si bien que, de même que l'Allemand ne saurait se penser en termes politiques, de même il ne saurait porter sur la musique un quelconque jugement de nature éthique. Car ce serait alors exprimer une marque de défiance, dont seul serait susceptible de faire preuve «le littérateur de civilisation ». Cette expression désigne le représentant par excellence de cette sphère méditerranéenne, façonnée par l'Europe des Lumières et adepte du strict logos, que le Thomas Mann de cette époque exècre : un ambassadeur de cette sphère qui se définit par des valeurs apparemment positives - elle se dit civilisatrice, morale, politique, et rationnelle - mais en lesquelles l'écrivain allemand croit déceler d'abord et avant tout le moyen de mener, via une propagande efficace, une entreprise de conquête et d'hégémonie. Soulignons ici qu'en forgeant une telle figure, Thomas Mann songe moins à tel ou tel écrivain français qu'à son propre frère Heinrich, exemple même de celui qui, selon lui, aurait trahi sa patrie spirituelle. On en trouve un équivalent fictif dans La Montagne magique (Der Zauberberg, 1924) en la personne de Settembrini qui, en un moment décisif, déclare :

Oui, je suis un amateur de musique - ce qui ne veut pas dire que je l'estime particulièrement, comme j'estime et j'aime par exemple le verbe, le véhicule de l'esprit, l'instrument, le soc étincelant du progrès. La musique, elle, est l'informulé, l'équivoque, l'irresponsable, l'indifférent. La musique est inappréciable comme moyen suprême de provoquer l'enthousiasme, comme force qui nous entraîne en avant et plus haut, lorsqu'elle trouve l'esprit déjà préparé à ses effets. Mais la littérature doit l'avoir précédée. La musique seule est dangereuse. La musique éveille le temps, elle nous éveille à la jouissance la plus raffinée du temps... elle éveille... et dans cette mesure même elle est morale. L'art est moral dans la mesure où il éveille. Mais, quoi, lorsqu'il fait le contraire? Lorsqu'il engourdit, endort, contrebalance l'activité et le progrès? Cela aussi, la musique le peut, elle sait à merveille exercer l'influence des stupéfiants. Une influence diabolique, messieurs. La drogue est du diable, car elle entraîne la léthargie, la stagnation, l'inactivité, la passivité, l'asservissement $[\ldots]^{\text {t9 }}$

19. Th. Mann, La Montagne magique [Fayard, I93I], Le Livre de Poche, 200I, p. I3I-I33. 
À l'inverse, Hans Castorp, le protagoniste du roman, trouve dans la musique, en l'occurrence un petit lied de Schubert, cette essence de la germanité en quête de laquelle il était lancé depuis le début de l'œuvre.

Mais plus le ralliement de Thomas Mann à la République et plus son hostilité au nazisme s'affirment et s'intensifient, plus l'écrivain apporte des correctifs et des infléchissements à son modèle germano-musical. Ceux que, naguère, il considérait comme de grands pédagogues es germanité, Schopenhauer, Wagner et Nietzsche, font désormais l'objet d'éclairages neufs et de réévaluations constantes. Sous sa plume, leurs figures, qualifiées tout à la fois de "grandioses" et de "souffrantes", deviennent de plus en plus ambivalentes. Par là, il tend à les soustraire des accaparements nationalistes auxquels il a pourtant lui-même souscrit autrefois. Sa grande étude sur Wagner ("Grandeur et souffrances de Richard Wagner" [Leiden und Grösse Richard Wagners", 1933]), en particulier, abondamment décriée par les nazis, lui vaut d'ailleurs un exil définitif. De plus en plus, Thomas Mann évoque et développe le thème d'une ambiguïté qui serait propre à la musique, et qui révélerait son essence diabolique. D'une certaine façon, l'écrivain semble désormais se ranger du côté de son Settembrini. Lorsque, à partir de 1943 à 1947, il entreprend son Docteur Faustus (Doktor Faustus, 1947), la musique y est hyperboliquement présentée comme "ambiguïté érigée en système (Musik die Zweideutigkeit ist als System). La marque même de la systématisation de l'ambiguïté musicale dans l'œuvre est son élaboration formelle extrêmement sophistiquée, qui mêle fantasme numérologique et référence dodécaphonique, afin que soit mise en scène une sorte de "démonisation" de l'histoire allemande dont, de Luther à Wagner et de Wagner à Hitler, la musique serait l'agent, le symptôme et la métonymie. "Je sentais que mon roman devait être ce dont il parlait» écrit Thomas Mann dans Le Journal du Docteur Faustus (Die Entstehung des Doktor Faustus, 1947) $)^{20}$ : là encore, c'est par la "musicalisation" du roman en vertu de principes «méloformels» que Thomas Mann rend compte des spécificités de la germanité - hier pour la glorifier, aujourd'hui pour revêtir son masque maléfique et la purifier.

Au terme de ce récit commencé avec Nietzsche, et engageant principalement la sphère germanique (prise au sens large du terme), on pourrait placer la figure de Thomas Bernhard, et les interrogations relatives à la "haute culture», quand l'histoire semble en avoir montré sinon l'ambiguïté substantielle, du moins les possibles compromissions. Avec lui, on peut d'ailleurs évoquer le cas des écrivains mélomanes autrichiens en général : la question du lien entre

20. La formule exacte est la suivante (Le Journal du Docteur Faustus, Bourgois, 1994, p. 6I : "Je sentais bien que mon livre devrait finir par être ce dont il traitait : une musique constructive.") 
identité autrichienne, Seconde Guerre mondiale, névroses contemporaines, et interrogations musico-littéraires, est en effet au centre des ouvres de Thomas Bernhard, Ingeborg Bachmann, Elfriede Jelinek ou Gert Jonke. Elle engage, à travers la musique, un procès générationnel et civilisationnel, qui n'est pas exempt de virtualités masochistes, puisqu'il s'agit, entre autres, de mettre à bas les idoles musicales, et tout le système de valeurs qui s'exprime à travers la célébration de la musique classique.

Enfin, à l'époque la plus contemporaine, la figure de Pascal Quignard illustre au mieux ce cas de l'amour-haine de la musique, tel qu'il peut se manifester chez les écrivains et philosophes mélomanes. À travers son œuvre, on peut en effet recenser deux conceptions antithétiques et complémentaires de la musique. Une vision d'abord négative, découlant de cette façon qu'aurait la collectivité d'être hypnotisée par la rumeur qu' elle dégage, de se fasciner ellemême par un bourdonnement sonore incessant. Selon Quignard, à travers une telle propension, particulièrement perceptible dans cette omniprésence du musical qui caractériserait le monde moderne, la collectivité viserait à dissimuler son origine animale en se donnant l'illusion de civilisation. En réalité, elle ne ferait qu'en dupliquer, sur le mode de la sublimation sonore, l'indépassable violence primitive. Par contraste, l'écrivain évoque la possibilité d'une relation positive à la musique : celle-ci permettrait en effet à l'individu de composer avec les mystères de son origine, et le sentiment de nostalgie qui lui est lié; en pacifiant, dans la lecture et l'écriture, conçues comme des activités de résistance, ce que le son garde de sa violence archaïque, elle rendrait possible l'authentique émergence de l'individualité.

Reprenons cela en détail. Fondamentalement, la collectivité, qui n'a eu de cesse de vouloir séparer l'homme et l'animal, ne se serait jamais détachée de son existence ancestrale de meute chasseresse. Dans toutes les inventions humaines, et particulièrement dans ses manifestations les plus apparemment civilisées, comme l'est la musique, on retrouverait un schème : celui du chant de veillée qui, sous le signe du chaman, anticipait ou concluait la chasse. Dès lors, tout pourrait être évalué à l'aune de ce plus petit dénominateur commun : le musicologue ne serait qu'un charognard; le quatuor à cordes européen rien d'autre que le rappel musical d'un très vieux rituel de mise à mort, etc. Et, croyant s'en détacher, la meute ne cesserait malgré tout de se retremper dans ce bain sonore, sorte de mise en commun des peurs fondatrices. Il va de soi que, dans cette masse anonyme, chaque individu abdique sa voix propre au profit de ce grand tout qui l'hypnotise. La série des traités qui constituent La Haine de la musique (1996) rabat ainsi l'ensemble des mythes, légendes et clichés musicaux que l'Orient et Occident n’ont cessé de formuler depuis la nuit des temps sur cet axe indépassable de l'animalité 
violente, égoïste, dominatrice de l'homme. Une animalité, surtout, qui perdurerait à travers tous les traits de civilisation, ainsi que le prouverait, à en croire Quignard, cette donnée extrême : la participation de la musique à la barbarie nazie. Participation et non pas simple présence : l'écrivain semble vouloir insister sur ce point. La musique, ainsi, ne serait plus rien d'autre que l'«expression sensible» de la détermination avec laquelle les hommes entreprendraient d'anéantir leurs congénères. La coprésence paradoxale, dans le monde contemporain, d'un bourdonnement sonore permanent, et d'un manque d'attention réelle accordée à la musique, simplifiée en quelque chose de brutal et de kitsch, s'expliquerait par ce biais : au moment même où le processus civilisationnel semble vouloir le plus sûrement la congédier, la meute ne ferait en réalité rien d'autre que de renouer avec la rumeur lancinante et assourdissante de son animalité première.

Qu'en est-il maintenant pour l'individu? Dans la ruche sonore de l'espèce, explique Quignard, l'individualité de chaque être est comme tétanisée. Dès lors, celle-ci ne peut se retrouver qu'à partir du moment où, échappant à la fascination, chacun fait de la quête de son "jadis", dans la musique et le silence, une activité de résistance. Dans ce cadre, l'individu ne se désolidarise en rien de l'espèce, mais il refuse de céder au caractère "tarabustant» des rythmes originels et des mélodies ancestrales qui continuent de s'exprimer à travers elle. Autrefois, la musique, qui était rare, représentait par excellence une activité propice à la quête de l'individualité et à son dévoilement; aujourd'hui, elle paraît repoussante parce qu'omniprésente. Quignard guette donc un autre univers musical que celui qui obsède ses contemporains. Plus : dans la retraite de l'écriture et de la lecture, au-delà de la musique, il dit aspirer au silence. Dans ce cadre, la littérature n'apparaît pas comme une pure négation du sonore; néanmoins, l'activité de l'écrivain s'apparenterait malgré tout, selon lui, à une "désobéissance - ce qui, au sens propre du terme, doit donc se comprendre comme un refus d'ouïr passivement le bourdonnement incessant du monde. Lire, et surtout écrire, c'est résister. Et le corollaire du silence est la solitude. La musique au bord du silence, le silence lui-même, c'est l'expression acceptée - et non pas niée, comme le fait l'espèce - du «jadis»; c'est le moyen le plus juste et le plus lucide de saisir le temps, le passé, la mort : trois données que la collectivité a de son côté tendance à vouloir refouler.

Boutès (2008), l'un des derniers traités consacrés par l'écrivain à la musique, affine cette dialectique. Quignard part de l'épisode des Argonautes dans lequel, chant contre chant, on voit Orphée lutter contre les sirènes, afin de protéger ses compagnons, lancés dans la quête de la toison d'or. Tout l'équipage en réchappe sauf le matelot prénommé Boutès, qui se jette à l'eau. 
Dans ce récit, deux musiques se font donc face : l'une, associée à Orphée, serait masculine, rythmée, liée à la pensée, à l'analyse, et à la collectivité; l'autre, apparentée aux sirènes, serait féminine, "continue», liée à la douleur, à la perdition, à l'individu. Les représentants de la première manifesteraient une peur à l'encontre de la seconde et des dangers que, selon eux, elle représenterait; et, dès lors, ils feraient tout pour l'endiguer et la tenir loin de la communauté. On voit bien ici l'ambiguïté inhérente à ces deux musiques : la première est apparemment moins dangereuse, mais elle serait en réalité belliqueuse, coercitive et potentiellement violente; la deuxième est essentiellement périlleuse, mais le danger qui lui est propre serait de grande valeur puisque, passant par-dessus le langage, et plongeant au cœur de la tristesse et de la douleur, elle permettrait la confrontation du moi avec l'inconnu en général, et les mystères de son être et de son origine en particulier ${ }^{21}$.

\section{Comment l'amour-haine de la musique peut devenir matière à fiction}

Il faut maintenant souligner que, dans le cadre de l'invention littéraire, l'amour-haine de la musique dont nous avons rendu compte ici peut faire l'objet de multiples mises en fiction : les écrivains rendent alors compte de différentes façons de ce triple défi musical défini plus haut, et dont le dénominateur commun, au côté de la fascination, est la réticence ${ }^{22}$.

Le "défi de l'infini» peut d'abord se faire dans un registre élevé (tragique, mythique, légendaire, etc.), et à des fins édifiantes. Cette propension est notamment caractéristique de l'approche romantique et postromantique. Dans ce cadre, l'esthétique fantastique apparaît en effet comme le moyen par excellence de transformer en fiction littéraire ce tiraillement existentiel qui caractériserait l'homme romantique, déchiré entre le fini et l'infini - un infini que la musique semble tout particulièrement prendre en charge. Un tel état tragique et douloureux peut être associé au thème du chant grandiose et terrifiant de la nature, ou à l'évocation d'un instrument ou d'un objet qui, dans des circonstances mystérieuses, s'animerait, et s'exprimerait de façon sonore ou chantante. L'indicibilité de la musique peut également être thématisée par

2I. Pour tout ce point, nous renvoyons à un article à paraître prochainement dans la revue Europe (A. Gefen, D. Rabaté [dir.]) : T. Picard, «La littérature contemporaine a-t-elle retrouvé un modèle musical? Le cas de Pascal Quignard.»

22. Dans le point qui suit, nous avons sensiblement remanié et affiné un premier essai de typologie proposé dans : T. Picard, "La littérature aime-t-elle vraiment la musique?», conclusion du volume Fascinations musicales, C. Dumoulié (dir.), Éditions Desjonquères, 2006, p. 267-283. Pour ne pas alourdir le présent article, nous renvoyons aux nombreux exemples littéraires que nous citions alors. 
l'image de la partition vierge ou de l'écriture musicale hiéroglyphique. Idole esthétique trompeuse, en particulier quand elle est cantatrice ou musicienne, automate chantant ou machine musicale : l'ambiguïté de l'infini musical peut également être rendue par l'entremise des thèmes du leurre et de l'illusion, voire de l'ensorcellement. Ce dernier trouve sa formulation la plus dramatique avec le motif de la "musique qui tue», soit que son effet soit effectivement mortel, soit que l'infini inatteignable auquel elle semble liée jette l'auditeur ou l'écrivain dans le désespoir et l'accule au suicide. De façon moins excessive, et couplée à la figure de l'artiste maudit tout à la fois élu et damné, la musique peut être associée à l'image de la marginalité, de l'isolement, de l'ascétisme, etc. Dans tous les cas se trouve alors à l'œuvre, comme envers du lieu commun du "concert céleste», une thématique musico-diabolique qui reprend certaines préoccupations présentes dès le Moyen Âge. De manière générale, il s'agit, par son biais, de mettre en fiction le caractère dérisoire de la destinée humaine en quête vaine, à travers la musique, de l'infini; ou, plus particulièrement au $\mathrm{Xx}^{\mathrm{e}}$ siècle, la question d'un mal de civilisation dont la musique pourrait être à la fois le symptôme et l'agent. Enfin, soulignons que si l'esthétique fantastique se déploie pour une bonne part dans le registre du sublime, elle peut également prendre, via cette thématique diabolique, une dimension grotesque. Le grotesque représente en effet le complément tragicomique du fantastique : il rend compte du même tiraillement entre fini et infini, mais se focalise sur la chute de l'homme hors de la sphère de l'infini et sur les effets comportementaux ridicules que cette chute hors de l'infini et la nostalgie de son royaume merveilleux peuvent engendrer.

C'est en effet que le deuxième type d'approche, qui fait glisser le défi de l'infini vers celui de l'éthique, a au contraire tendance à se faire sur le mode de la démythification. C'est-à-dire qu'il a pour effet (et parfois pour fin) de désacraliser l'idéal musical. Il est alors plutôt caractéristique d'un traitement de type naturaliste ou décadentiste. On ne s'étonnera donc guère de trouver, parmi les lieux communs d'écriture formulés dans ce cadre, la comparaison de la musique avec l'alcool, la drogue et, plus largement, l'association avec un imaginaire de la maladie, qu' elle soit mentale (par exemple la monomanie ou toute sorte de folie qui peut d'ailleurs parfois mener au crime), ou physique (voir l'association de hasard ou de nécessité, établie dans certains récits naturalistes et décadentistes, avec la tuberculose ou la syphilis; ou encore la mise en relation, dans le récit fantastique, entre un chant inouï et une complexion maladive). En prolongement évident de cette thématique, on trouve le motif de la musique qui stérilise, particulièrement représenté dans la littérature fin de siècle à travers de multiples figures d'esthètes et de dilettantes mélomanes. Si, en liant procréation et création, l’imaginaire sexuel rejoint ici 
les questions de poétique, c'est que les champs de la biologie, de l'art, et de la morale ont, à cette époque, particulièrement tendance à converger. On en veut pour preuve l'association récurrente de la musique avec un visage d'Éros considéré comme douteux. Car - là aussi, faux hasard et, souvent, vrai lien de nécessité -, le caractère suspect que représente, aux yeux de certains écrivains, la jouissance musicale, s'agrège à une représentation de ce qui, dans le cadre de la fiction, avec caution ou non du narrateur, est considéré comme une perversion sexuelle : érotomanie, homosexualité, pédophilie, inceste, sadomasochisme, fantasme androgynique, fétichisme, etc. Et, dans ce cadre, une certaine propension consistant à fétichiser la voix se révèle particulièrement récurrente.

On comprend alors que l'imaginaire musical puisse régulièrement se trouver mêlé aux grands récits décadentistes et autres visions du monde à caractère "déclinologique», tels qu'ils ont pu se développer depuis la fin du $\mathrm{XIX}^{\mathrm{e}}$ siècle. On recense en effet, chez les écrivains mélomanes comme chez les penseurs mélosceptiques, la rémanence d'un récit philosophico-historique constitué par les trois composantes suivantes : âge d'or, chute, rédemption. Et si la musique est fréquemment placée du côté de la rédemption, elle est plus souvent encore considérée comme un symptôme, voire comme un agent de la décadence. Plus on avance dans le temps, plus, dans les scènes ou séquences d'évocation musicale, s'impose le récit canonique de l'envol de l'âme dans le ciel lumineux de l'extase musicale, puis de sa chute sur un sol terrestre enténébré, ou dans un océan infini dans laquelle elle ne peut faire autrement que se noyer. Et, dès lors, il devient également commun, dans les discours, de considérer que la musique serait, à un moment ou à un autre, entrée dans l'ère de l'impureté, qu'elle aurait progressivement hypertrophié ses moyens, et pratiqué une surenchère de l'expression au détriment d'une éthique de la forme. C'est pourquoi, à rebours de cette certitude décadentiste, on s'emploie à quêter, toujours plus en amont dans l'histoire de la musique, un hypothétique âge d'or datant d'avant la décadence. En outre, cette vision décadentiste de l'histoire de la musique qu'entretiennent écrivains et penseurs mélomanes vient très souvent nourrir une interrogation sur le devenir de la culture et de l'histoire en général. Pour ce faire, on utilise alors de façon privilégiée le genre de la "fiction achronique», qui consiste à faire dialoguer, par-delà le temps et les styles, de grandes figures et, tout particulièrement, de grands musiciens, pour créer par leur intermédiaire des confrontations entre sphères musico-spirituelles diverses, en vue d'une synthèse et, le plus souvent, d'un bilan pessimiste de la culture européenne.

Le troisième type d'approche engage plus strictement le défi éthique et tient pour une bonne part à la question - problématique d'un point de vue 
méthodologique - de la récupération de la musique et de son utilisation à des fins politiques. On l'a vu avec le cas extrême du traité antimusical à la Quignard, l'horizon d'un tel imaginaire est évidemment la catastrophe de la Seconde Guerre mondiale, précédée de la "crise de l'esprit». En la matière, le camp de Terezín, où la musique occupa une place privilégiée dans l'activité de propagande et le système concentrationnaire nazis, tient lieu de paradigme. Dans ce cadre, les fictions qui associent musique et barbarie, musique et kitsch mortifère, ou musique et totalitarismes, sont nombreuses - au chef desquelles on trouve bien sûr celles qui sont plus directement liées au nazisme, pris dans sa globalité, ou dans le seul cadre des camps de concentration. Notons que se développent également aujourd'hui les fictions qui font de l'entrée dans l'ère $\mathrm{du}$ "tout sonore» un des signes de ce "totalitarisme mou» qui, selon certains écrivains, serait la conséquence du déclin des idéologies et du triomphe de l'«imagologie» dans les sociétés occidentales avancées. Dans un cas comme dans l'autre, de tels thèmes peuvent prendre la forme de romans de sciencefiction ou d'anticipation à caractère "dystopique».

Il faut enfin évoquer notre dernier défi, d'ordre intersémiotique. L'impossibilité de "dire» la musique en littérature peut en effet passer par certaines modalités agressives de mises en fiction, qui prennent alors la forme d'un programme de reconquête. Parmi celles-ci, on peut tout d'abord citer le genre du «tombeau musico-littéraire». Ici et là, les écrivains semblent en effet avoir pris un certain plaisir à représenter des scènes de mises à mort ou simplement de décès de musiciens. On le comprend peu à peu : s'ils composent un tel thrène, c'est très manifestement dans le but de rivaliser avec la musique, de prendre le relais, et de montrer leur puissance. Dans la littérature récente, les écrivains ont cependant de plus en plus tendance à orchestrer le deuil de la littérature en même temps que celui de la musique. Dans un même ordre d'idée, le signe de l'impossibilité de parler du chant en littérature peut avoir pour conséquence la récurrence d'un motif instructif, celui de la fragmentation (du continuum musical) ou du morcellement (du corps du chanteur), signes que, de la jouissance vocale, l'écriture ne semble pouvoir recueillir qu'un résidu. Le thème de la machine musicale (gramophone, poste radio, etc.) est quant à lui placé sous le signe mortifère du tombeau : malgré son désir prométhéen d'éterniser la jouissance musicale, l'écrivain n'en conserverait que quelque chose d'inanimé et dangereux. En vertu d'une habitude consistant à doter les arts d'un sexe, la rivalité entre musique et littérature peut encore être allégorisée par le biais de la lutte des sexes, à la fois dans les essais et dans les fictions, où les personnages peuvent ainsi devenir les allégories de tel ou tel art, lancé dans une lutte avec tel ou tel autre. Enfin, on a vu que le grotesque pouvait représenter un moyen de contourner le défi de l'infini musical; il peut également servir, de façon plus ou moins bienveillante, 
à se venger de son indicibilité. C'est le cas avec la caricature ou la parodie exercées à l'encontre du personnel musical (compositeur, chanteur, musicien, et surtout amateur); ou encore avec la tentation de réécrire de façon démystifiante une fable musicale portée par le grandiose et le sublime. De telles inclinations possèdent une certaine ambiguiité : on ne sait s'il s'agit, à travers elles, de rendre compte, non sans agressivité, d'une forme d'impossibilité littéraire, ou de mettre en garde contre les dangers du sublime.

\section{Complément et correctif : de la haine de la musique en général au rejet de telle ou telle musique en particulier, jeux de modèles musicaux}

Rappelons ceci : plus on avance dans le temps et se rapproche de notre époque, plus la vision que l'on a de la musique se complexifie : si les œuvres dont parlent les écrivains dans leurs discours sont d'abord essentiellement contemporaines, la musique est de plus en plus amenée à se constituer en histoire, dotée d'une certaine profondeur de champ. Ce qui veut dire que l'on peut la segmenter en macro-périodes esthétiques et historiques, en mouvements, et qu'il devient également davantage possible, en raison de caractéristiques esthétiques distinctes, de jouer ceux-ci les uns contre les autres.

Parmi les principales lignes de partage que l'on peut distinguer, citons l'opposition ancienne et structurelle entre musique vocale et musique instrumentale ou - ce qui ne revient pas au même - entre musique à programme et musique absolue. Il faut également évoquer les oppositions entre esthétiques nationales, nourries par des propos relevant de "l'imagologie»: ce sont les confrontations entre la France et l'Italie durant la période classique, et entre l'Italie et l'Allemagne à la période romantique - complétée par une multiplication des revendications et tensions identitaires à la fin du XIX ${ }^{e}$ siècle et au début du $\mathrm{xx}^{\mathrm{e}}$ siècle. Rappelons ici tout de même que ces oppositions ne sont pas purement d'ordre identitaire : elles renvoient aussi, en profondeur, à une certaine façon d'envisager la musique et, notamment, à la relation que doivent par exemple entretenir, dans une œuvre mixte, le vocal et l'instrumental, ou encore les différentes façons qu'il y a de musicaliser et de vocaliser un texte. Sachant que telle et telle de ces façons se voit en retour justifiée par la référence aux spécificités supposées de tel ou tel génie national.

Ce peuvent être enfin les oppositions entre macro-périodes ou entre grandes esthétiques homologuées : il peut par exemple s’agir de jouer le baroque, le classicisme ou la modernité contre le romantisme; la musique savante contre la musique populaire; la musique classique contre le jazz ou le rock, etc. Ceci peut évidemment être affiné : il est ainsi possible d'opposer un premier 
baroque ou un premier romantisme à un second, etc. Ajoutons que ces lignes de partage s'exercent souvent par un jeu d'oppositions entre grandes figures chargées d'incarner hyperboliquement telle ou telle identité nationale (Verdi ou Debussy contre Wagner) ou telle ou telle esthétique (Bach ou Mozart contre Wagner). Concluons en rappelant que ces oppositions structurelles ont des conséquences directes sur la littérature, puisqu'elles impliquent des choix non seulement de thèmes, mais encore de styles ou de formes. Et qu'elles dessinent également une axiologie complexe, autour de valeurs comme la grâce, la profondeur, la légèreté, la virtuosité, etc. 
